\title{
Entwicklung, Methoden und Aufgaben der Verkehrsgeschichte
}

\author{
Teuteberg, Hans Jürgen
}

First published in:

Jahrbuch für Wirtschaftsgeschichte, Jg. 1994, Heft 1, S. 173 - 194, Berlin 1994

Münstersches Informations- und Archivsystem multimedialer Inhalte (MIAMI) URN: urn:nbn:de:hbz:6-06429445480 


\title{
Entwicklung, Methoden und Aufgaben der Verkehrsgeschichte
}

\author{
Von Hans-Jurgen Teuteberg (Manster)
}

\section{Zum Begriff der Verkehrsgeschichte und ihren Erkenntniszielen}

Als Adam Smith 1776 bei seiner "Untersuchung über die Natur und Ursachen des Reichtums der Nationen" entgegen der physiokratischen Lehre nicht den Boden, sondern die Arbeit eines Volkes als eigentliche Quelle allen Wohlstandes bezeichnete und auf das Prinzip der Arbeitsteilung hinwies, von dem die Ergiebigkeit des Faktors Arbeit abhänge, lenkte er zugleich einschränkend den Blick darauf, daß diese Produktivitätssteigerungen nicht überall und ungehemmt stattfinden können, weil sie durch unterschiedliche Absatzchancen beschränkt werden.' Das ókonornische Wachstum stehe nämlich in einer bestimmten Relation zum Warenaustausch auf den Märkten. Die Entwicklung der Wirtschaft in den letzten zweihundert Jahren hat den großen Vordenker moderner Nationalökonomie in diesem Punkt voll bestätigt: Alle Produktionssteigerungen der sich industrialisierenden Wirtschaftszweige sind seit dem späten 18. Jahrhundert Anlaß wie Resultat der Verbreiterung der Absatzverhältnisse gewesen.

Die wachsende Nachfrage nach Wirtschaftsgütern ist ohne gleichzeitige Steigerung der Verkehrsleistungen nicht denkbar. Ohne die entscheidenden Verbesserungen im Güter-, Personen- und Nachrichtenverkehr wäre selbst bei gröBter ökonomischer Freiheit nicht einmal die Hebung des kleinräumigen Binnenhandels, geschweige denn die Entwicklung zum weltweiten internationalen Warenaustausch möglich gewesen. Die Umgestaltung der herkömmlichen Transportverhältnisse und ihre Anpassung an die Erfordernisse der modernen Volkswirtschaft können daher in ihrer Bedeutung kaum überschätzt werden. Der mit dem Beginn des "Dampfzeitalters" sich erstmals aus den Schranken der organischen Natur befreiende und seitdem immer mehr beschleunigende, seine Kapazität, Regelmäßigkeit und Sicherheit stark vergrößernde und die Frachtkosten stark senkende Verkehr schuf eine der wesentlichen Voraussetzungen für die von dem schottischen Moralphilosophen Adam Smith so scharfsinnig erkannte, immer weiter fortschreitende Differenzierung von Produktion und Konsumtion.

Die nachfolgenden Ökonomen, aber auch die Staats- und Gesellschaftswissenschaftler haben, solchen grundlegenden Einsichten zustimmend, das Verkehrswesen dann unter den verschiedenen Aspekten immer wieder untersucht und auch auf der gedanklich abstrahierenden Ebene theoretisch zu durchdringen versucht. Der Wirtschaftswissenschaftler Andreas Predöhl hat fast hundertjährige Überlegungen zusammenfassend hierzu festgestellt, der Verkehr umfasse im weiteren Sinne alle möglichen Formen sozialer Kontakte und ermögliche so die Konstituierung menschlicher Kultur. In der engeren Bedeutung bezeichne er "die Gesamtheit aller Vorgänge, die im Bereich der Wirtschaft der Raumüberwindung dienen, nämlich dem Transport von Personen, Gütern und Nachrichten in jeder Form zu Wasser, zu Lande und in der Luft". ${ }^{2}$ Der Transport wurde von ihm als eine Spielart der 
Produktion begriffen; denn die Umwandlung eines konsumfernen in ein konsumnähere Wirtschaftsgut sei ebenso entscheidend wie die veranderung eines Rohstoffes in ein Halbbzw. Fertigfabrikat. Die Ortsveränderung kann somit als Prämisse für jede sich spezialisièrende Wirtschaft und damit für die daraus hervorgehende Hebung des Lebensstandards angesehen werden. Ergänzend läßt sich noch hinzufügen, daß der Verkehr letztlich die Basis für alle Versuche darstelit, die wirtschaftichen Ungleichheiten auf dieser Erde alimähich auszugleichen. Obne ihn ist die Beseitigung des großen Gefalles zwischen den reichen Industriestaaten und det armen Entwicklungsländern nicht zu erreichen

In neverer Zeit ist ferner darauf hingewiesen worden, dab die rasch fortschreitende Verbilligung und Qualitätssteigerung des Personenverkehrs die jahrhundertealten Bindungen des Menschen an seinen Heimatort erheblich gelockert haben. ${ }^{3}$ Das Aufkommen der modemen Massenverkehrsmittel führte wur Zentralisierung der Produktion, dem Anschwellen der städtischen Bevölkenung und einer folgenschweren Trennung der beiden Lebenssphären Wohnen und Arbeiten. Diese Strukturwandlungen haben bekanntich zu groben Anpassungsproblemen und sozialen Konflikten geführt.

Auf der anderen Seite entfalteten die immer dichter werdenden Verkehrsnetze aber auch integrierende Wirkungen. Diè verkehrsmäßige Erschließung sehr unterschiedlicher Territorien brachte nicht nur eine ähnlich parallele Verteilung der Wirtschaftsgüter, sondern auch staaticher und geselischafticher Dienstleistungen im Sinne einer verbesserten Daseinsvorsorge. Der Verkehr fübrte, etwas zugespitz formuliert, zu einer Identifikation de Individuums mit großßeren räumlichen Einheiten und beförderte so die Bildung von einheitlichen Volkswirtschaften und damit zugleich auch von Nationalstaten.

Aber der moderne Verkehr hat wie jeder technische Fortschritt ein Janusgesicht: Die Anhänger einer möglichst ungehinderten Freihandelspolitik, die stets eifrige Anhänger eines Verkehrsausbaus waren, haben seit dem 19. Jahrhundert immer wieder darauf verwiesen, daß freier Handel und Verkehr tendenzieli volkerverbindend und friedenssciftend wirken. Aber diese zu optimistische Fortschritsthese erscheint am Ende des 20. Jahrhunderts strittig, da die modernen Verkehrsmittel auch die Formen der Kriegführung erweitert haben und häufig gerade nicht konfiktminimierend wirken.

Die steigende Zahl der Verkehrsunfalle, die erhöhte Inanspruchnahme der natürlichen Ressourcen durch zunehmenden Energieverbrauch und die wachsende Umweltbelastung durch Lärm und toxische Schadstoffe sind Schattenseiten des revolutionāren Anstiegs der Verkehrsleistungen. Die vom modernen Verkehr ausgehenden Belastungen sind keineswegs alle neu, aber erst in den beiden letzten Jahrzehnten als dringliche und von der Politik $z$ lossende Probleme erkannt worden. Die okklogischen Aspekte des Verkehrs wurden von den älteren Definitionen noch nicht erfaßt und müssen hier noch eingebracht werden.

Bei der Behandlung der modernen Verkehrsproblenxe spielt die Verkehrsgeschichte eine schon lange erkannte wichtige Rolle. Sie allein kann erklären, wie die gegenwärtigen verkehrspolitischen Probleme entstanden sind und welche Lossingsversuche frühere Generationen datür angeboten haben. Zudem wurzeln manche Formen des heutigen PersonenGüter" und Nachrichtenverkehrs in älteren Techniken und Verfahren, die zun Vermächtni menschlicher Kultur gehören und gelegentliche Aktualisierungen verdienen. Was sind nun

\footnotetext{
W. Hamm, Art. "Verkehr", in: Handwörterbuch der Wirschaftswissenschaften, Bd.8. Stutgant 1980,
}

Entwoklung. Methoden und Aufgaben der Verkehrsgeschiche

die generellen Aufgaben eines Verkehrshistorikers? Zunächst einmal hat er gemäß der historisch-wissenschaftlichen Methode alle theoretisch deduzierten Aussagen, besondar solche der Sozial* und Wirtschaftswissenschaften, anhand der überlieferten historischen Zeugnisse empirisch zu uberprüfen und die nicht falsifizierten Fakten in den Gang der allgemeinen Geschichte tinzuordnen, d.h. mit den bereits bekannten Ereignissen der Vergangenheit systematisch in Bezug zu setzen. Diese Aufgabe ist dem Historiker eigentümlich und nicht delegierbar, da die Vertreter anderer Disziplinen nur die Entwicklung ihres eigenen Faches und daher eng begrenzte Ausschnitte aus der Vergangenheit zu liefern vernögen. Der Historiker benutzt dabei die Methode des historischen Vergleichs. Zu seinen primären Aufgaben gehört es, den Wust des Geschehens in der Vergangenheit zu strukturieren und prägende Formveränderungen in möglichst typologischer Weise zu beschreiben. Die Verkehrsgeschichte hat mit anderen Worten die Aufgabe, die historisch wesentichen Ereigruisse und Gestalten herauszustellen und nach historisch einschnetdenden Zasuren zu suchen. Diese müssen keineswegs immer mit den politischen Wendepunkten ubereinstimmen. Eine umfassende Geschichte des menschichen Verkehrs im Sinne einer Raumäberwindung von Personen, Gütem und Nachrichten ist auf langen Strecken eine allmählich ablaufende Strukturgeschichte und läst sich nicht allein an einzelnen technischen Innovationen oder Personen festmachen.

\section{Entstehung und Entwicklung der verkehrsgeschichtlichen Forschung}

Nach diesen Prolegontena ist nach der Entstehung, dem Gang und heutigen Stand der verkehrsgeschichtlichen Forschung zu fragen. Ein Uberblick wird dadurch erschwert, daß für das deutschsprachige Gebiet Mitteleuropas keine alle Aspekte urmfassende Bibliographie existiert, so daß man auf die zahlreichen, weit verstreuten kleinen Literatursammlungen zu einzelnen sachlichen, râumlichen oder zeillichen Ausschnitten greifen mußs. Die beste Yorarbeit bietet immer noch hier das Standardwerk von Fritz Voigt "Der Verkekr" aus dem Jahre 1965. Die dort verzeichnete Literatur gliedert sich in die Abschnitte: 1. Allgemeine Geschichte des Verkehrs, 2. Verkehrsgeographie, 3. Verkehr im Altertum, Mitielalter, Merkantilismus (16.-18. Ih.) und im Zeitalter der Industrialisierung (seit 19. Jahnrhundert), 4. Spezifische Probleme der Verkehrsgeschichte, 5. Verkehrspolitik, 6. Spezifische Probleme der Verkehrspolitik, 7. Fremdenverkèh, 8. Akuelle Probleme der Verkehrswissenschaft.

Wie diese Gliederung zeigt, entspricht sie nicht mekr den gewachsenen Ansprüchen einer modernen Verkehrswissenschaft und vor allem nicht einer historisch orientierten Disziplin Die Literaturliste vermag nicht za befriedigen, handelt es sich hier doch fast ausschließlich um mehr oder weniger neue Sekundärliteratur; die historischen Quellen zur Verkehrsgeschichte, vor allem aus früheren Jahrhunderten, sind nur ganz fragmentarisch erfakt und auch nicht zuveriässig zitiert. Wenngleich die großartige gelehrte Leistung des Verkehrswissenschaftlers Fritz Voigt aicht verkleinert werden soll, so muß aber doch festgesteltt werden, daß dieses Kompendium nur als eine Vorarbeit für eine umfassende Aufarbeitung der geschichthichen Verkehrsverhältnisse anzusehen ist. Verstandlicherweise muß auch die stark angewachsene Literatur der letzten füntundzwanzig Jahre nachgetragen werden.

4 F. Voigt, Verkehr Bd. 2, 1. und 2. Hätfe: Die Entwicklung der Verkehrssysteme, Berfin 1965. 


\section{Erste Versuche zur Erfassung der Entwicklung der Transportverbältisisse}

Versucht man dennoch ohne veriäliche bibliographische Basis die Genese und Entfaltung der verkehrsgeschichtichen Forschung im Umriß zu skizzieren, dann ergibt sich etwa folgendes Bild: Da sich bereits Schriftsteller der griechisch-förnischen Antike, des hohen und späten Mittelalters sowie der frühen Neuzeit mit dem Transport von Gütern, Personen und Nachrichten lebhaft beschäftigt baben und die in allen groBen Bibliotheken und Archiven ruhenden Quellenmaterialien nur in eng begrenzten Ausschnitten zu verkehrsgeschichtlichen Fragen ausgewertet wurden, läß sich der Beginn der engeren verkehrsgeschichtlichen Untersuchungen noch nicht exakt bezeichnen. Soweit der Historiker bisher erkennen kana, gibt es offenbar dafür verschiedene Wurzeln: Bei der Zusammenstellung von Apodeniken, Itinerarien und Reiseberichten, aber auch bei der Abfassung von Staatsbeschreibungen und der Herausgabe von Enzyklopädien okonomisehen und technischen lohalts fuhlten sich Autoren aus verschiedensten Antässen inspiriert, erste auch historisch informierende Informationen über gewisse Teile des Waren-, Personen- und Nachrichtenverketirs zu veroffentlichen. Dabei hat offenbar die Post als erste wichtige staatliche Transportorganisation im Heiligen Römischen Reich deutscher Nation seit dem 17, Jahrhundert besondere Beachtung gefunden." Aber auch die Entwicklung der Scbiffahrt auf Flüssen und Kanälen sowie auf dem Meer wurde frühzeitig anhand von Quellen dokumentiert. $\mathrm{Da}$ die gelehrten Schriftsteller in der klassischen Literatur bewandert waren, darf es nicht verwundern, wenn sie sich of besonders den antiken Verkehrsverhältnissen zuwandten. Die historisch nicht genau überprüften und oftrnals halblegendären Nachrichten über frühere Verkehrsverhälnisse können freilich nur als erste Vorläufer eloer wissenschaftichen Verkehrsgeschichte angesehen werden. Einige maßgebende Merkantilisten und Staatswissenschaftler wie z. B. Wilhelm von Schröder, Johann Gottlieb von Justi und Samuel Freiherr von Pufendorf, ferner Johann Christian Lünig, Johann Jakob Moser und Veit Ludwig von Seckendorff, aber auch Johannes Becknanin, Karl Friedrich Anton, Wilhelm August Schlözer und Georg Heinrich Zink, vor allem aber der Göttinger Statswissenschaftler und Kameralist August Heinich Ludwig Heeren mit seinem Werk "Ideen ibber Politik, den Verkehr und den Handel der womehmsten Volker der alten Welt" sind $\mathbf{z} . \mathbf{B}$, im 17, und 18.

5 Der kenntaisreiche Verkehrshistoriker F. C. Huber teilt mit, über die Entwicklung des Verkethrs und besonders der Post habe es schon vor Ablauf des 17. Jahrhunderts über 60 Abhandiungen gegeben. Wenggieich diese micht raher belegte Angabe anhand einschlägrger Xataloge (z,B. in der Herzag-Augusr-Bibiothek in Wolferbutrel) noch nachzuprufert wärs, so erscheint diese Schätzung keneswegs ais unstrunis, sondem eher wohl noch zu gering. Vgl. F.C. Huber, Die geschichliche Entwicklung des modernen Verkehrs, Tübingen
1893 , S.II. Vgl. femer als Beispista fruher post- und strafiengeschichtlicher Abhandlungen Ch. Leonhardi, Scriplores et excerpta de regali posiarum jure, Leipzig 1710;A.F. Lueder, Strafsenbau und Wegegeschichte,

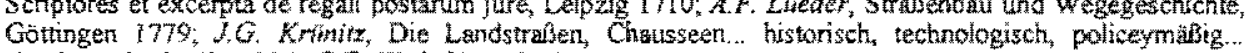
abgehandelt, Berlin 1804; CF. Wrebeking, Praktische Anleinung zur Aufrihrung, Wiederherstellung und Erhaltung bequemer und den Commerz fordernder LandstraBen, Leipzig 1804. Die Beschäftigung mit antiken Straben- und Postvertaltnissen murde offenbar durch das weit verbreitete Werk eines Iranzösischen Aufklärers befordert; $N$. Bergier, Kistoire des grands chemins de l'Empire romain... 2 Bde., Brüssel 1728 oder 1736

6 Vgl. z.B. P.J. Marperger, Neueröffnete Wasserfahrt auf Plüssen und Canälen, Dresden 1723; J.L. Hogrewe, Beschreibung der in England seit 1759 angelegten und jetzi grötentheils voliendeten schiff-
baren Kanăle, zur innem Gemeinschaft der vornehmsten Handelssiädte. Nebst einem versuch einer Geschichte der inlundischen Schiffahrt und alter bis jezr in und auRerhalt Europas bekannten Kañle Hannover 1780. $J J$ Berghaus, Geschichte der Schiffahrshonde bei den vornetmeten voikern des Alter thums, 2 Bde., Leipzig 1792 .
Jahrhundert mit verkehrsgeschichtichen Notizen hervorgetreten. "Wenngleich Heeren noch nicht über die Methoden einer modernen Geschichtswissenschaft verfügte so gehört er doch ohne Zweifel zu den "Gründungsvătern" einer Verkehrsgeschichtsschreibung, die im 17/18. Jahrhundert unter dem Einfuß des Naurrechts und der Aufklärung ihre ersten Regungen zeigte. Sie bildete freilich zunächst nur ein buntes Sammelsurium von mehr zufälig ausgegrabenen Nachrichten, die unsystematisch vermengt rit anderem Wissensstoff dargeboten wurden.

2. Die Begründung der modernen Verkehrswissenschaft durch Friedrich List und die Historische Nationalökonomie

Den eigentlichen Aufschwung fir eine Beschäftigung im engeren Sinne gab es freilich erst im 19. Jahrhundert unter dem Historismus, der die eigentlichen Grundlagen für eine moderne Geschichtswissenschaft geschaffen hat. Aus der Fülle der nun schnell anwachsenden Literatur können hier nur einige wenige Namen herausgegriffen werden. Neben dem bayerischen Oberbergrat Joseph von Baader, dem böhmischen Ingenieur Franz Anton Gersiner, dem westfalischen Unternehmer Friedrich Harkort und dem rheinischen Kaufnarn und Bankier David Kansemann hat sich bekanntlich vor allem Friedrich List erstmals mit dem neuen Verkehrsmittel, der Eisenbahn, in Deutsckland beschäftigt und ist in seinen zahlreichen Schriften auch auf den Verkehr in seiner gesamten Entwicklung eingegangen. In seinen groben Werken, aber auch in seinen zablreichen kleineren Aufsătzen und Lexikonartikeln hat er die verkehrswissenschaftliche Forschung, basierend auf seinen amerikanischen Erfahrungen, stimuliert und ihr neue Wege gewiesen." Wenngleich der schwäbische Gelehrte und Politiker keineswegs der erste war, der die weittragende Bedeutung des au Schienen laufenden "Dampfwagens" erkannte und sein Einflu $B$ auf die Anfänge staatlicher Eisenbahnpolitik nicht überschätzt werden darf, so gebührt ihm doch ein erster Ehrenplatz in der Verkehrsgeschichtsschreibung, da er trotz seiner vielfachen speziellen Projekte stets zum Allgemeingültigen überging und bewut den historischen Vergleich suchte. Dabei kam er zu Ergebnissen, die noch niemand vor ihm so formuliert hatte. So machte er sich Gedanken uber den ökonomischen und gesellschaftichen Nutzen des neuen Verkehrsmittels, über

7 A.LH, Heeren, Ideen abber die Pohik, den Verkehr und den Handel der wonehmsten Volkar der alten Welt. 3 Bde., 2.Aufl, Göttingen 1805-1812. Die übrigen Titel sind verzeichnet bei Voigi, Verkekr (wie Amm, 4). Die hier angegebenen Werke müBten freilich auf eire Beschäftigunis mis der Verkehrsgeschichte noch einmal gründlich durchgeseken und die bereffenden Stellen gerau bezoichnet werden. Für Kenner der frihneuzeitlichen Literatur besteht kein Zweifel, dâf es sich hier um eine Zufallsauswahl handelt und sich die Zahl der einschlägigen Werke noch stark vermehren labt. Dis eirschlagigen historischen Biblographien zwischen dem 16. Und 19. Jahrh

F. List Das deuische National-Transport System ir

let, Alooa 1838; ders. Über ein sāchsisches Eisentalks- uød staatswirthschaftlicher Beziehung beleuchEisentahnsysterns und insbesonders über die Anlegung einer Eisenbabn von Leipzig nach Dresden, Lejpzig 1833; ders., Art. "Eisenbahn und Kanale", in: C. wan Rolteck C. Welcher (Hg), Stats-Lexikon oder Encyklopaedie der Statswissenschaften, Bd. 4, Attona 1837, S. 650-778; ders, Das deutsche Eisenbahnsystem als Mittel zur Vervolikommnung der deutschen Industrie, des Deutschen Zolivereins und des deutschen Nationalverbandes überhaupt, Snugart 1841. Friedrich List schrieb noch zahtreiche weitere Aufsätze in dem von ihm herausgegebenen "Eisenbahn-Journal" (Braurschweig 1835-1837). Eine genaue Auflistung aller seiner einschiggigen Arbeiten in F. List, Schritten, Reden, Briefe, Bd. 3: Schriften Verkebrswesen, Berlin 1930 Vgl. v.a. H. Getring. Friedrich List, Leipzig 1956; W.O. Henderson, 
seinen Einfluß auf die Produktions- und Arbeitsteilung sowie die Preisbewegungen auf den Märkten. Dabei wurde er nicht müde zu betonen, daß der Massenguterverkehr das eigentliche Merkmal der Eisentahn sei, weshalb hier der Personenverkebr zurücktreten müsse, der früher den Landtransport beherrscht babe.

Sein leidenschaftiches Eintreten für das neue Verkehrsmittel machte ihn aber nicht blind für die Bedeutung anderer Verkehrsmittel, z.B. für die Rolle des neuen Dampfschiffes bei der Seeschiffahrt. An die historische Entwicklung anknüpfend wies er auf die große Rolle der Bimnenschiffahrt hin und sah den newen Schienentransport oftmals nur in Ergänzung des traditionellen Wasserstraßensystems.

List propagierte überdies als einer der ersten eine sinnvolle Arbeitsteilung der verschiedenen Verkehrsträger genäß den unterschiedichen Anforderungen an Schnelligkeit, RegelmäBigkeit und Frachtkosten. Selbst die gesellschaftlichen und politischen Folgen der Eisenbahn in Kriegs- und Friedenszeiten, besonders die Eröffrung neuer Kulturwelten für den Personenverkehr gerieten in sein Blickfeld, wobei durch die ständigen Vergleiche mit der Vergangenheit die Revolutionierung der bisherigen Transportmôglichkeiten ihm erstmals voll bewubt wurde.

Mit List beginnt daher nicht nur die moderne Verkehrswissenschaft in Deutschland, sondern zugleich auch die systematische verkehrsgeschichtliche Betrachtung. Wenngleich der große Schwabe kein Historiker im heutigen Sinne war und die Geschichte ihm mehr zur Abstützung seiner handels- und verkehrspolitischen Argumente diente, so bleibt es doch erstaunlich, wie frïhzeitig er das Ende der alten und den Begin der neuen Verkehrswell im Industriezeitalter ahnungsvoll beschrieben hat. Welche uberragende Stellung List hier zugewiesen werden muB, erkennt man am besten, wenn man ihn mit anderen verkehrswissenschafthchen Schriftstellern seiner Zeit vergleicht. Auch diese beschätigten sich zwar mit der Einwirkung der Dampfmaschine auf den Verkehr und saunmelten erste Zahlenangaben und technische Hinweise, aber der unwäzende Charakter des neuen Verkehrsatitalters blieb ithen doch noch weitgehend verborgen.

Noch schärfer und wissenschaftlich auch praziser als List, der in erster Linie auf die praktischen Auswirkungen seiner Schriften bedacht sein mußte, äuberte sich in der Mitte des 19. Jahrhunderts dann Karl Knies als typischer Vertreter der nun auftauchenden Historischen Schule der Nationalökonomie. In seinen beiden glänzend formulierten Werken "Die Eisenbahnen und ihre Wirkungen" (1853) und "Der Telegraph als Verkehrsmittel" (1857) brachte or das Wesen der neuen und alten Verkehrsmittel erstmals in eine feste theoretische Verbindung und verglich die Einfuhrung der Dampfkraft in Verkehr mit dem ahnlichen Vorgang in den Fabriken. Die weitreichende historische Bedeutung beider Innovationen stellte or zu Recht auf dieselbe Stufe. Wichtiger als die Schnelligkeit der Eisenbahn, die seine Zeitgenossen vornehmich faszinierte, war fur in die Frachtkostandegression, insbe-

9 Vgl. C.F. Nebenius, Der deutsche Zolverein, sein \$ystem und seine Zukunft, Carlsnuhe 1835; LF.E. Lotz, Handbuch der Staatswirthschaftsletre, 3 Bde., 2 . Aufl., Erlangen 1837; J von Fiadier, Über die Einfihitrung der Kunststraßsen (Eisenbahnen) in Deutschland, Leipzig 1836; D. Honsemasm, Die Eisen

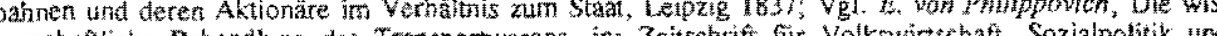
senschaftiche Behandlung des Transpo
Verwalture $14,1905, \mathrm{H}, \mathrm{S}, \mathrm{S}, 1 \mathrm{18}$

$10 \mathrm{~K}$. Kniger Die Fisenbahn und thre Wirkungen, Braunschweig 1853: ders. Der Telegraph als Verkehrsmittel. Mit Erörterungen ther den Nachrichtenverkehr uberhaupt, Tubingen 1857. sondere bei den wertgeringen Massengütern. Knies erkannte femer, daß die Verbesserung der Transpormittel mit Hilfe der Dampfmaschine einen "latenten Verkehr" schafft, d.h. daß die modemen Transportmöglichkeiten auch neuen Verkehrsbedarf erzeugen. Auch die neue RegelmäBigkeit der Raumüberwindung, die dem Gesez des neuen "Maschinenzeitalters" folgte, wurde von ihm als neue einheitliche Zeitbestimmung begriffen, womit der so ziokulturelle Aspekt der Verkehrsrevolution herausgestelt wurde.

Knies, der sich auch mit den Wirkungen des neuen Transportsystems auf die Bodenpreise, die landwirtschaftliche und gewerbliche Produktion sowie die herkömmliche Handelsorganisation, den Kapitalbedarf und das Städtewachstum beschäftigte und schlieblich den Beginn eines ganz neuen Kommunikationszeitalters mit dem Telegraphen beraufziehen sah, hat damit über List hinausgehend die Rolle des gesamten Verkehrs für die Wirtschaft, das Rechtsleben der Völker, die Politik des States und sogar die Volkserziehung behandelt. Es gibt kaum einen Aspekt des Verkehrs, den or übersehen hătte. Selbst die Probleme des kinftig wachsenden Nahverkehrs in den großen Städten wurden von iho bereits hellsichtig erkannt. Als maßgeblicher Mitbegründer der Historischen Schule der Nationalökonomie legte er Wert darauf, alle theoretischen Anaahmen durch die Fakten der Geschichte und Statistik ai überprüfen.

Nach Knies sind andere Vertreter der Historischen Schule der Nationalökonomie wie Wilhelm Roscher, Ernst Engel, Max Sering und vor allem Gustav Schmoller dieser von ihm eingeschlagenen Linie weiter gefolgt und haben nun einzelne Aspekte des Verkehrs gründlicher untersucht." Wahrend der in Leipzig lehrende Wilhelm Roscher eine langfristige Beziehung zwischen Verkehrs- und Bevölkerungsentwicklung herzustellen versuchte, ging der erst in Strabburg und dann in Berlin wirkende Schmoller zuerst der Wirkang der Eisenbahnen auf die traditionelle handwerkliche Guterproduktion nach, wobei er die These vertrat, dab erst die neuen Transportmittel den wirklichen Gegensatz zwischen dem alten Zunftsystem und der liberalen Gewerbefreiheit offenbart hätten. Die damals weithin nur in Rechtsproblemen befangene Debatte über die Vor- und Nachtelle der neuen liberalen Wirtscbaftsordnung wurde dadurch auf einen ganz neuen Boden gestellt. Schmoller machte auch erstmals durch seine Schriften klar, daß durch die masserhafte Heranbringung konsumfertiger Guter, die in einem festen Laden verkauft werden, das alte Versorgungssystem der Städte, das auf zünftigen Privilegien, Märkten und Messen beruht hatte, nun unwidernuflich verändert werde. Nicht die Industrieausstellungen wie Karl Knies noch irrtumlich geglaubt hatte, sondern der emporwachsende Einzel- und Großhandel sowie die Warenborsen veränderten, gestützt auf den neuen Massentransport, die jahrhundertealte städtische Infrastrukhur. Immer wieder rückte Schmoller mit der thm eigenen höchst anschaulichen Gegeruberstellung von Fakten und Zahlen aus Vergangenheit und Gegenwart den gewalkigen

11 W. Roscher, System der Volkswirthrschaft, Bd. 3: Nationalokonomik des Gewerbefieibes und des Handels, Stuttgart 1881; ders, Uber Kornhandel und Teurerungspolitik, 3. Aufl., Stuttgart $1852 ; O$. Schmoller, Zur Geschichte der deutschen Kleingewerbe, Halle 1870; ders., Der moderne Verkehr im

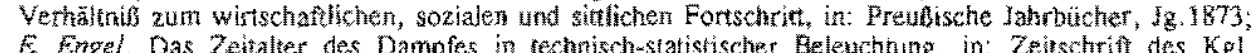
E Engel, Das Zeitalter des Dampfes in rechnisch-statistischer Beleuchtung, in: Zeitschrift des $\mathrm{Kgl}$.

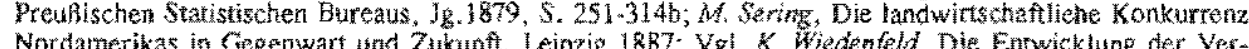
kehrsmittel und die landwirtschatiliche Konkurrenz des Auslandes im letzten Menschenalter, in: Zeitschrift fir Agrarpolitik $2,1904, \$, 1-26$ 
Wandel ins Blickfeld, der durch die modernen Verkehrsmittel in Gang gesetzt worden war Für ihn hatte nach einem später vielzitierten Gedanken der Verkehr durch eine bessere Warenverteilung die Aufgabe, an der Stelle einer kleinen armen Hütte ein neues gewaltiges Wohnhaus für die Menschen zu errichten und auch die sonst benachteiligten Keller- und Mansardenräume behaglicher zu machen. Er rückte damit die Auswirkungen der Ver kehrsmittel auf die Verteilung und die Konsumtion in den Vordergrund, da die Produk tionsprobleme hinsichtlich des Transports und Absatzes nun einer Lösung zustrebten. Der große Nationalökonom und Historiker formulierte erstmals auch eine Stufentheorie für den Gang der Verkehrsentwicklung. So unterschied er drei wesentliche Epochen: 1. Die ältere Zeit des zufälligen primitiven Nachbarschaftsverkehrs. 2. Die Periode des bescheidenen, aber nun regelmäßig werdenden Lokalverkehrs. 3. Den Eintritt in das Zeitalter des großen Massenverkehrs, der die Erdteile und Völker verbindet. In seinem vielgelesenen "Grundriß der Volkswirtschaftslehre" (1900-1904) hat er seine Stufentheorie näher erläutert, gegen die, wie gegen alle solche Periodisierungsversuche, heute allerdings viel einzuwenden ist. ${ }^{12}$

Auch neben ihm gab es bedeutende Einzelleistungen anderer Wissenschaftler. So legte der zuerst in Dresden, dann in Berlin wirkende Statistiker Ernst Engel erstmals größeres Zahlenmaterial für die Verkehrsgeschichte vor, den revolutionären Umbruch des 19 . Jahrhunderts nun auch quantitativ sichtbar machend. Er stützte sich dabei auf eine Fülle einschlägiger Statistiken, die unter anderem von Carl Friedrich Wilhelm Dieterici, Friedrich Wilhelin Freiherr von Reden und Alfred Bienengräber zuvor zusammengetragen worden waren 13 Der bekannte Agrarpolitiker Max Sering stellte dagegen internationale Preisvergleiche an, die die ungeheure Wirkung des Massengütertransports am Beispiel der Getreidemärkte demonstrierten. Damit wurde ein gewichtiger Beitrag zu der um 1900 heftig geführten Debatte über Agrar- und Industriestaat erbracht, die sich um die Einfuhr amerikanischen Weizens und die Beibehaltung der Agrarschutzzölle drehte. ${ }^{14}$

In der zweiten Hälfte des 19. Jahrhunderts ist es dann unter dem Einfluß der schnel akkumulierenden Erkenntnisse über die Entwicklung der Verkehrsverhältnisse auch zu ersten Versuchen gekommen, das vorhandene Wissen zu bilanzieren und erste Monographien zur Verkehrsgeschichte vorzulegen. Dabei bildeten sich mehrere Gruppen mit unterschiedlichen Intentionen heraus: Zunächst gab es Autoren, die sich in Fortführung aufklärerischer Traditionen der älteren Kulturgeschichte und Altertumskunde weiterhin verpflichte fühlten. Die Archive deutscher Städte durchstöbernd, sahen sie in erster Linie ihre Aufgabe

2 G. Schmoller, Grundriß der Volkswirtschaftslehre, Bd. 2, 7.-12.Tsd. erg. und verm. Teil 2, München 1919 , S.1-43, bes. S. 5

13 Die im 19. Jaht Su ebracht Vgl aus der Fülle der Titel $x$ B. CFW. Dielerici, Statistische Übersicht über die wichnigsten Gegenstände des Verkehrs und Verbrauchs im Preußischen Staate und im Deutschen Zollverbande in dem Zeitraume von 1831 bis 1836 , Berlin u.a. 1838, funf Fortserzungen 1842-1857; F. W. [Frhr.] von Reden Erwerbs- und Verkehrs-Statistik des Königstaates Preußen. In vergleichender Darstellung, 3.Abtl. Darmstadt 1854; ders., Deutschland und das übrige Europa. Handbuch der Boden-, BevölkerungsErwerbs- und Verkehrsstatistik, des Staatshaushalts und der Streitmacht, 2 Abtheilungen, Wiesbaden 1854; A. Bienengräber, Statistix des Verkehrs und Verbrauchs im Zollverein für die Jahre 1842-1864
nach den veröffentlichten amtlichen Kommerzialübersichten dargestellt, Berlin 1868 .

14 Die neueste Zusammenfassung dieser Problematik und aller einschlägigen zeitgenössischen Schriften, die für die internationale Verkehrsgeschichte von großem Nutzen sind, bringt jetzt M. Steinkiuhler, Agraroder Industriestaat? Die Auseinandersetzungen um die Getreideha
Reiches. In: Europäische Hochschulschriften Bd. 529, Berlin 1992. darin, die Leistungen des gesellschaftlich wie wirtschaftlich emporkommenden deutschen Bürgertums im Mittelalter und der frühen Neuzeit herauszustellen. So entstanden wichtige Studien zur Handels- und Verkehrsgeschichte früherer Jahrhunderte, aber auch noch einmal zur antiken Welt. ${ }^{15}$ Eine andere Gruppe versuchte dagegen, von den damaligen Streitfragen ausgehend, gerade den Umbruch des Verkehrs durch die kapitalistische Wirtschaftsordnung in den Vordergrund zu stellen. ${ }^{16}$ Eines der vielgelesensten Bücher ist in diesem Zusammenhang das kleine Büchlein von Walther Lotz "Verkehrsentwicklung in Deutschland 1800 1900" gewesen, das, aus populären Vorträgen hervorgegangen, bis 1920 vier Auflagen erlebte. Nimmt man sich diese Schrift einmal näher vor, dann erkennt man, daß dieser Schüler Lujo Brentanos ganz im Bann des liberalen Fortschrittsoptimismus gestanden hat. Nach einem knappen Rückblick auf die vorindustriellen Verkehrsverhältnisse wird breit und emphatisch der Durchbruch des Eisenbahnwesens und die daraus folgende Güter- und Personentarifpolitik geschildert. Ein kleineres Kapitel ist der Binnenschiffahrt gewidmet, während zum Schluß die Wirkungen der modernen Verkehrsmittel auf alle Zweige der Volkswirtschaft zusammengefaßt werden. Die reichen Literaturhinweise nach jedem Kapitel sind Zeichen dafür, auf welche zahlreichen Einzeluntersuchungen, Handbuchartikel und Fachzeitschriften der Verfasser sich stützen konnte. ${ }^{17}$ Die meisten der von ihm zitierten

15 E. Curtius, Zur Geschichte des Wegebaus bei den Griechen, Berlin 1855; G. Schaefer, Ursprung und Entwicklung der Verkehrsmittel. Nach geschichtlichen Quellen dargestellt, Dresden 1890; E.E. Hudemann, Geschichte des rómischen Postwesens während der Kaiserzeit, Kiel 1866, 2. Aufl. Berlin 1878; ders., Das Postwesen der romischen Kaiserzeit, Theil 1: Die äussere Geschichte desselben, Kiel 1866; $E$ Harrmann, Entwicklungs-Geschichte der Posten von den ältesten Zeiten bis zur Gegenwart mit besondere Beziehung zu Deutschland, Leipzig 1868: H. von Stephan, Verkehrsleben im Alterthum, in: Historisches Taschenbuch, Jg.9, 4.Folge, Leipzig 1868, Sp.1-136; Ritter, Die Reichspost der römischen Kaiser

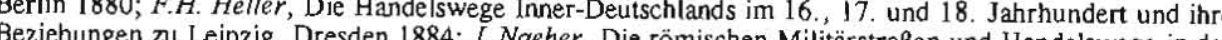
Schweiz und Südwestdeutschland, insbesondere in Gassner, Zum deutschen Stralienwesen von der ätesten Zeit bis Tur Mitte des 17., Jhalburg 1888, 1889; O. Wanka, Edler von Rodlow, Die Brennerstraße im Altertum und Mittelalter, Prag , Leipzig Rauers, Zur Geschichte der alten Handelsstraßen in Deutschland Versuch einer quellenmäßigen Übersichtskarte. Mit 4 kartogr. Beilagen. Hrsg. vom Verein für hansische Geschichte, Gotha 1907; A. Schulte, Geschichte des mittelalterlichen Handels und Verkehrs zwischen Westdeutschland und Italien mit Ausschluß von Venedig, 2 Bde., Leipzig 1900; P.H. Scheffel, Verkehrsgeschichte der Alpen, 2 Bde., Berlin

6 Zum neuen Verkehrsmittel Eisenbahn äußern sich u.a. Schreiber, Die preußischen Eisenbahnen und ih Verhältnis zum Staat 1834-1874, Berlin 1874; V. [Frhr.von] Röll u.a. (Hg.), Eneyklopädie des gesammten Eisenbahnwesens in alphabetischer Anordnung, 7 Bde., Wien 1890-1895, 2. Aufl. in 10 Bden., Wien 1912-1923; $H$. Schwabe, Geschichtlicher Rückblick auf die ersten 50 Jahre des preußischen EisenbahnStuttgart 1895. Die Verwaltung der öffentlichen Arbeiten in Preußen 1900-1910. Bericht an Seine Majestät den Kaiser und König erstattet von dem Minister für öffentliche Arbeiten, Berlin 1901 (enthäl ausführliche Kapitel über alle Verkehrsarten, besonders aber über den Eisenbahnverkehr). 1901 (enthäl Die Entstehung der Rheinischen Eisenbahn-Gesellschaft 1830-1844, Essen 1910. Über die Verbesserungen der Binnenschiffahrt berichten z.B. L. Sympher, Transportkosten auf Eisenbahnen und Kanälen, Berlin 1885; G. Schanz, Die Mainschiffahrt im 19. Jahrhundert und ihre künftige Entwicklung Bamberg 1894; ders., Der Donau-Main-Kanal und seine Sehicksale, Bamberg 1894; H. Schwabe, Die Entwicklung der deutschen Binnenschiffahrt bis zum Ende des 19. Jahrhunderts, Berlin 1899; L Sympher, Die Zunahme der Binnenschiffahrt in Deutschland von 1875-1895, Berlin 1899; G. Eger, Die

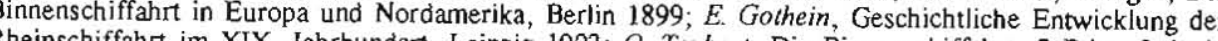
Bde., Leipzis 
Autoren waren, wie man unschwer erkennen kann, Anhänger der historischen Nationalökonomie. Kein Wunder, wenn sie, wie etwa das Beispiel des Schmoller-Schülers Georg Schanz lehrt, oftmals weit in die Geschichte ausgriffen und von der Statistik regen Gebrauch machten. In der dritten Auflage des berühmten Handwörterbuches der Staatswissenschaften ist bei den Artikeln "Binnenschiffahrt", "Kanäle", "Schiffahrt", "Eisenbahnwesen", "Transport", "Post", "Telegraphie und Telephonie", "Verkehrsmittel" und "Verkehrswege" den wirtschafts-, sozial- und kulturgeschichtlichen Betrachtungen breiter Raum eingeräumt worden; allein der Entwicklung des Verkehrswesens im Mittelalter wurden 32 Spalten gewidmet. ${ }^{18}$ Hier sind auch die Ergebnisse der zeitgenössischen Forschung über den Straßen-, See- und Nachrichtenverkehr, die bei Lotz nicht berücksichtigt wurden, übersichtlich zusammengefaßt worden.

Resümierend läßt sich feststellen, daß von List über Knies bis zu Schmoller und Lotz ein einheitliches Bemühen sichtbar wird, den Zusammenhang der Verkehrsmittel aus der historischen Entwicklung heraus zu begreifen und nach ihren Ursachen zu fragen. Allen Autoren ging es in erster Linie darum, die notwendigen Fakten herbeizuschaffen und das Wesen des Verkehrs in seinem größeren politischen, gesellschaftlichen und kulturellen Zusammenhang zu sehen. Eine Wende in der Methodologie bedeutete es aber, als 1878 der Wiener Gelehrte Emil Sax in einem bedeutenden zweibändigen Werk "Die Verkehrsmittel in Volks- und Staatswirthschaft" das Wesen des Transports, zu den Prinzipien der klassischen englischen Wirtschaftslehre zurückkehrend, ausschließlich auf deduktivem Wege erörterte. ${ }^{19}$ Die ganze geschichtliche Entwicklung wurde von ihm dagegen in einen kleinen Anhang verwiesen. Der von dem Österreicher Carl Menger später ausgelöste Methodenstreit in der Nationalökonomie wurde hier bereits sichtbar.

\section{Die Fortbildung der Verkehrstheorie durch Emil Sax}

Sax wollte nicht wie die Historische Schule der Nationalökonomie die Entwicklung des Verkehrs erforschen, sondern dessen Position im System der damaligen Volkswirtschaf exakt bestimmen. Von hier aus sollten dann die Grundsätze einer staatlichen Verkehrspolitik entworfen und festgelegt werden, wie die Verkehrseinrichtungen optimal zu gestalten seien. Ihn interessierten mit anderen Worten nicht die Ursachen, sondern allein die Auswirkungen des modernen Verkehrslebens. Damit schuf er das erste deutschsprachige Werk, das eine allgemeine Theorie der Verkehrswissenschaft aufstellte. In rein gedanklich-abstrahierender Weise untersuchte er allein am Beispiel der Eisenbahn in scharfer Scheidung der Massengüter von den gewerblichen Erzeugnissen die Preissenkung und den Preisausgleich, Bd. 15), 2. erw. Aufl. 1906, 4. Aufl. 1920. Weitere erste Zusammenfassungen der modernen Verkehrs-
revolution des 19 . Jahrhunderts waren F.C. Huber, Die geschichtliche Entwicklung des modernen Ver-
kehrs, kehrs, Tübingen 1893; $R$. van der Borght, Das Verkehrswesen, Leipzig 1894; F. Ulrich, Staatseisenbah-
nen, Staatswasserstraßen und die deutsche Wirtschaftspolitik, Leipzig 1898; F. C. Philippson, Handel und Verkehr im 19. Jahrhundert, Berlin 1899; G. Cohn, Zur Geschichte und Politik des Deutschen FinanzVerkehrs- und Verwaltungswesens, Sturtgart 1905; G. Biedenkapp, Die Entwicklung der modernen Verkehrsmittel, Berlin 1911

18 F.C. Huber, Art. "Verkehrsmittel", in: Handwörterbuch der Staatswissenschaften, Bd. 8, 3. Aufl., Jena 1911, Sp. 171-194; T. Sommerlad, Art. "Verkehrswesen im Mittelalter", in: ebd., Sp. 194-209. Vgl.

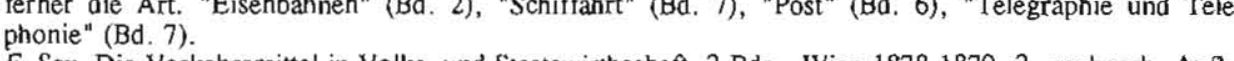

19 E. Sax, Die Verketron

3 Bde., Berlin 1918-1922. die sich bei der Verbesserung der modernen Transportmittel bei gleichbleibendem Verhält nis von Angebot und Nachfrage ergaben. Sax sah in der ständigen Verbesserung des Transportapparates einen der Hauptgründe für die Entwicklung zum industriellen Großbetrieb, weil sich die durch den verbesserten Verkehr sinkenden Produktionskosten und die gleichzeitige Ausweitung der Absatzräume wechselseitig antrieben. Sax konnte mit seiner Betonung des Wertes der Massengüter das Wesen der Weltmärkte und die weltweite geographische Arbeitsteilung nun besser erklären. Durch die modernen Verkehrsmittel, so stellte Sax richtig fest, werde die jahrhundertealte Gunst der natürlichen Verkehrslage nun durch den Besitz von Arbeits- und Kapitalkräften überflügelt. Damit wurde das alte Thünensche Gesetz, das noch auf der vorindustriellen Marktlage basierte, durchbrochen.

Sich an den Erfahrungen seiner Zeit orientierend, wies der Wiener Gelehrte einschränkend aber darauf hin, daß sich aus dem Transport von Massengütern mit Hilfe moderner Verkehrsmittel noch nicht automatisch eine Preissenkung ergebe. Diese Wirkung trete vielmehr erst dann ein, wenn der Verkehr in regelmäßige feste Tarife eingebunden werde, wobei sich die Frachthöhe nach dem Güterwert und den Entfernungen richte. Der Absatz eines Gutes hänge damit nicht von der absoluten, sondern der relativen, d.h. am Wert des Produktions- und Konsumtionsortes gemessenen Frachthöhe ab. Die angemessenen Tarife als Teil der Verkehrspolitik erschienen ihm darum letztlich wichtiger als der Bau neue Verkehrsmittel. Wenngleich Sax noch nicht die Funktion internationaler Frachtvereinbarungen und eines festen überseeischen Linienverkehrs kannte, deckte er erstmals ein anderes ökonomisches Gesetz auf: Wegen der relativ hohen Kosten stehen die Gesamtverkehrskosten mit der Intensität des Verkehrs im umgekehrten Verhältnis bzw. mit anderen Worten bestimmen bis zu einer gewissen Grenze bei modernen Transportmitteln nicht die Transportkosten den Marktpreis, sondern umgekehrt der Marktpreis die Transportkosten Damit ergibt sich die zu beobachtende Tendenz zur Bildung von Staatsmonopolen, Preiskartellen oder doch staatlich regulierten privaten Verkehrsunternehmen.

Andere Volkswirte haben diese systematischen Überlegungen weiter fortgeführt und z.B., wie es Adolph Wagner tat, auf das öffentliche gemeinwirtschaftliche Element in den Verkehrsmitteln hingewiesen, was als Argument für die Verstaatlichung der Eisenbahn diente. ${ }^{20}$ Auch wurde, auf Sax zurückgehend, von ihm die These vertreten, daß die Intensität einer Bodennutzung durch den Verkehr den allgemeinen Kulturzustand des betreffenden Gebietes determiniere. Daraus folgerten andere aber umgekehrt, daß die Vielgestaltigkeit eines Verkehrsnetzes den Grad des erreichten Wirtschafts- und Kulturzustandes widerspiegele.

Die von Emil Sax eingeleitete Abwendung vom ökonomischen Historismus darf aber nicht dahin verstanden werden, als sei nun überall sofort eine Abwendung von der Verkehrsgeschichte bei den Nationalökonomen erfolgt. Ganz im Gegenteil kam es bei verkehrshistorischen Untersuchungen zu neuen Höhepunkten. Zunächst legte Werner Som

20 Adolph Wagner hat als erster die Tatsache hervorgehoben, daß Eisenbahn - Aktiengesellschaften als kapitalistische Großunternehmungen öffentliche Elemente in sich aufgenommen haben und nach einer gcwissen staatlichen Regulierung verlangen. Nur bei einer staatlichen Eisenbahn könnten die Frachten nach dem volkswirtschaftlichen Allgemeinnutzen ausgerichtet werden. Vgl. A. Wagner, Finanzwissenschaft, 3 Teite, Leipzig 1877-1889 u.ö. $(=$ Lehr- und Handbuch der politischen Okonomie Bd. II); ders.

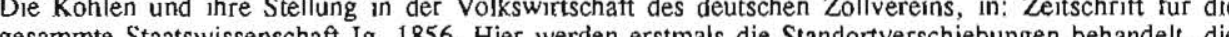
die Eisenbahn durch den billigen Transport von Steinkohlen bewirkt. 
bart sein vielbändiges, anfänglich heftig umstrittenes Mammutwerk "Der moderne Kapitalismus" vor, das er dann aufgrund der Kritik noch einmal gründlich überarbeitete. ${ }^{21}$ In mehreren großen Abschnitten schilderte er dort, auf viele Jahrhunderte und ganz Europa ausgreifend, unter genauer Zitierung eines üppigen Quellenmaterials den Gang der gesamten Verkehrsentwicklung in verschiedenen Ländern, wobei er immer wieder auf interessante Querverbindungen aufmerksam machte. Er verstand es auch, die gewaltigen Stoffmassen mi Hilfe geschickt gestellter leitender Fragestellungen und umfassender Begriffsbildungen übersichtlich zu gliedern. Über seine Terminologie läßt sich gewiß manchmal streiten, doch erscheint sie stets gedanklich anregend. Den Begriff Verkehr hat er wie folgt aufgegliedert (Siehe Schaubild: Werner Sombarts Definition des Begriffes Verkehr um 1900).

Schaut man sich das Kapitel "Verkehrswesen" im zweiten Band seines Werkes "Der moderne Kapitalismus", der dem europäischen Wirtschaftsleben im Zeitalter des Frühkapitalismus im 16. 17. und 18. Jahrhundert gewidmet ist, mit seinen über 200 Druckseiten einmal näher an, dann muß man dem Autor bescheinigen, daß er alle Seiten der Verkehrsproblematik in der frühen Neuzeit beleuchtet hat. Selbst so abgelegene Thesen wie Seeversicherung, das ältere Herbergswesen und erste Formen von Geschäftsanzeigen als Vorformen moderner Wirtschaftswerbung werden unter sorgfältigem Verweis auf die historischen Quellen erörtert. Dabei bleibt es manchmal mangels ausreichender Forschungen allerdings bei Zufallsnotizen, deren Repräsentativität schwer zu erkennen ist. Sombar erörtert aber meist die Qualität einer Quelle und charakterisiert so ihren Erkenntniswert. Wie Schmoller hat er auch zum Mittel der Stufentheorie gegriffen, um die Masse der historischen Fakten in eine Systematisierung zu bringen. ${ }^{22}$

Der Autor hat sich daneben auch noch speziell dem 19. Jahrhundert zugewandt und in seinem immer wieder aufgelegten Bestseller "Die deutsche Volkswirtschaft im neunzehnten und zu Anfang des 20. Jahrhundert" auch dort der Verkehrsgeschichte besondere Beachtung geschenkt. ${ }^{23}$ Das ungeheuer dicht und spannend geschriebene Werk, das alle Zweige de Verkehrs behandelt, gehört ebenfalls zu den Meisterleistungen der Verkehrsgeschichtsschreibung vor dem Ersten Weltkrieg.

Die Blütezeit der verkehrsgeschichtlichen Forschung hat auch einige Nachbardisziplinen nicht unbeeindruckt gelassen. Hatten schon Albert Schäffle und Lorenz von Stein bei ihren verfassungs- und verwaltungsrechtlichen bzw. gesellschaftswissenschaftlichen Studien auf

21 W. Sombarl, Der moderne Kapitalismus, 2. Aufl,. Bd. 2: Das europäische Wirtschaftsleben im Zeitalter des Frühkapitalismus vornehmlich im 16., 17. und 18. Jahrhundert. München 1917, S. 231-420.

22 Sombart unterscheidet $z$. B. drei Epochen im Wegebau: Die erste reicht bis zur ersten Hälfte des 17. Jahrhunderts; bis dahin reicht das alte mittelatterliche Straßensystem, jetzt beginnen erste Bestrebungen zur Verbesserung der Hauptstraßen in den größeren Territorialstaaten. Die zweite Epoche reicht vom späten 17. bis zum frühen 18. Jahrhundert, wo der Straßenbau durch den absolutistisch-merkantilistischen Staa erstmals systematisch und energisch in Angriff genommen wird. Die technischen wie finanzietlen Mitte reichen jedoch noch nicht aus, um ein verzweigtes Straßennetz zu schaffen und zu unterhalten. Erst in der riode pin die dann direkt in 1 . Jither des "Hochkpitismu" bzw. riode ein, die dann direkt in das Ze $S$. Darmstadt 1991 (= Wege der Forschung Bd. CL XXVI); H. Kellenbenz, Art. "Wirtschaftsstufen", in Handwörterbuch der Sozialwissenschaften Bd. 12, Stuttgart 1962, S. 260-269.

23 W. Sombart, Die deutsche Volkswirtschaft im neunzehnten und im Anfang des 20. Jahrhunderts, Berlin 1909. Hier zitiert nach der 7. Aufl. 1927, S. 238-275.

Schaubild 1: $\quad$ Werner Sombarts Definition des Begriffes Verkehr um 1900

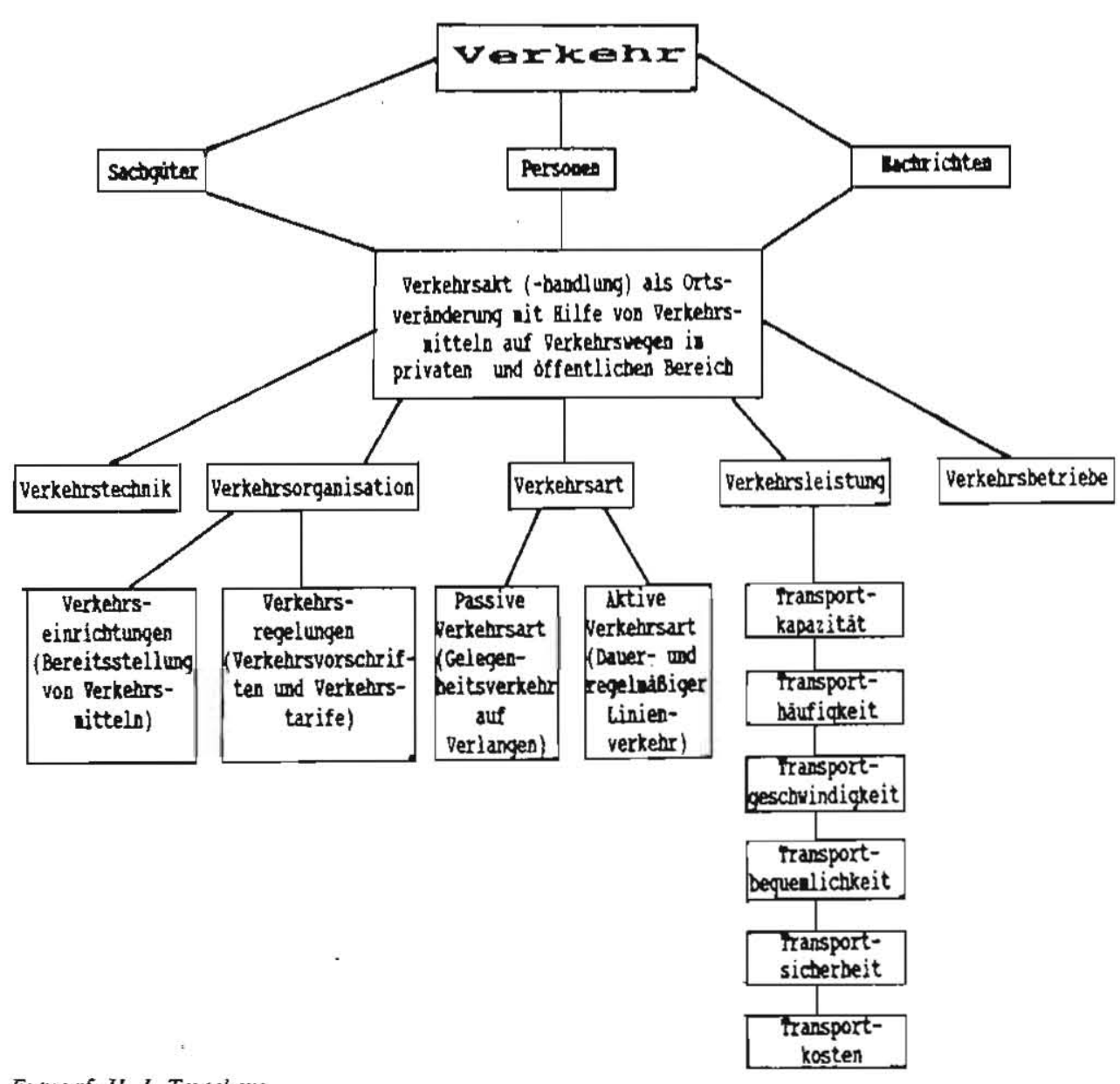

Entwurf: H.J. Teuteber

die ungeheuren Auswirkungen der modernen Verkehrsrevolution aufmerksam gemacht24, so sah sich dann Max Weber veranlaßt, bei seinen weitgespannten Untersuchungen über die "Entfaltung des kapitalistischen Geistes" dem Warentransport im Rahmen von Handel und Geldverkehr gebührende Beachtung zu schenken. ${ }^{2 s}$ Die Resultate der Forschungen der Historischen Schule aufarbeitend, gelang es ihm hier, zu generellen Erklärungen menschlicher Raumüberwindung vorzustoßen. So erfährt der Leser bei ihm z B., daß Seehandel tund Seeraub in langen Jahrhunderten überhaupt nicht $z u$ trennen waren und sich die Funktionen

24 L. von Stein, Handbuch der Verwaltungslehre, 2. Aufl., Stuttgart 1876, S. 348-428; A. Schäffe, Bau und Leben des socialen Körpers, Bd̛. 3, Tübingen 1878, S. $163 \mathrm{ff}$.

25 M. Weber, Wirtschaftsgeschichte (1919), 3. Aufl., Berlin 1958, S. 174-190, S. 251-255 
eines Handels- und Kriegsschiffes erst allmählich getrennt haben. Ferner schildert er die Vorteile eines frïhen Karawanenhandels und das Wesen der antiken Proxeni, d.h. der heute seltsam anmutenden Symbiose von Gastfreundschaft und kaufmännischer Interessenvertretung, die noch weit bis ins Mittelalter hineinwirkte.

\section{Der Beitrag der Verkehrsgeographie}

Auch die Geographie hat sich unter dem Eindruck des Historismus der Geschichte der Verkehrsmittel zugewandt und sie unter ihren spezifischen Aspekten zu studieren begonnen. Johann Georg Kohl, Friedrich Ratzel, Alfred Hettner, Wilhelm Goetz, Richard Henning und Freiherr von Richthofen haben hier interessante Studien vorgelegt. Darin fragte man z.B. nach dem Zusammenhang zwischen Bevölkerungsansiedlung und Verkehrsausbreitung, dem Einflu $\beta$ der Transportmittel auf die lokale Populationsverteilung oder nach dem Entstehen der Hauptwege des Weltverkehrs. ${ }^{26}$

Alfred Hettner hat 1897 die seit der Mitte des 19. Jahrhunderts in Gang gekommenen Arbeiten für eine Verkehrsgeographie erstmals in ausführlicher Form zusammengefaßt und die Aufgaben und Methoden einer solchen Fachrichtung beschrieben. ${ }^{27}$ Ähnlich wie bei der Entwicklung der Verkehrsgeschichte sieht er in ihr zunächst ein unsystematisches Zusammentragen zufällig erlangter Nachrichten geschichtlichen, ökonomischen und technischen Inhalts, die mit dem engeren Anliegen der Disziplin nur indirekt zu tun haben. Dann entwickelt sich eine rein praktische oder angewandte Verkebrsgeographie, welche die Tatsachen des Verkehrs als gegeben hinnimmt und nur nach den Folgewirkungen für die Zukunft fragt, um die Verkehrsverhältnisse im Sinn eines Kulturfortschritts zu verbessern. Obwohl schon Karl Ritter als Hauptbegründer einer wissenschaftlichen Geographie auf die Rolle von einigen Tieren als Transportmittel in Exkursen aufmerksam machte, war es jedoch Johann Georg Kohl vorbehalten, erstmals konsequent wie die Historiker nach den Ursachen der Verkehrsmittel und Verkehrsorganisationen zu fragen. Kohl faßte, eine erste Theorie für die räumliche Ausbreitung von Verkehrsnetzen aufstellend, den Verkehr als eine mechanische

26 Vgl. zur Entwicklung der Verkehrsgeographie, die an dieser Stelle nur kurz gestreift werden kann, u.a. J.G. Kohl, Der Verkehr und die Ansiedelungen der Menschen in ihrer Abhängigkeit von der Gestaltung der Erdoberfläche, Dresden 1841; B. von Cotta, Deutschlands Boden, sein geologischer Bau und dessen Einwirkungen auf das Leben der Menschen, Bd. 1, Leipzig 1854, S. 18ff.; $K$. Jansen, Die Bedingtheit des Verkehrs und der Ansiedelungen der Menschen durch die Gestaltung der Erdobernläche nachgewiesen insonderheit an der Cimbrischen Halbinsel, Kiel 1861; K. Andree, Geographie des Welthandels, 3 Bde., Wien 1862-1877, 2 Auf., Stuttgart 1877, vollst. neu bearb. Aufl. hg. v. Franz Heldrich, Robert Sieger, Stürmer a. Mestaltung sämmtlicher Eisenbahnnecze Sturmer, Geschichte der Eisenbahnen. Entwicklung und jetzige Geshangraphie. Eine Darstellung des der Erde, 2 Bde., Bromberg 1872-1876; $M$ modernen Weltverkehrs mit besonderer Berücksichtigung der Eisenbahnen, Stuttgart 1875; F. Ratzel, Die geographischen Bedingungen und Gesetze des Verkehrs und der Seestrategik, in: Geographische Zeitschrift 9, 1903; F. Frhir. von Richthofen, Vorlesungen über allgemeine Siedlungs- und Verkehrsgeographie, bearb. u. hg. v. Otto Schlüter, Berlin 1908; R. Henning, Die Hauptwege des Weltverkehrs, Jena 1913; ders., Verkehrsgeschwindigkeiten in ihrer Entwicklung bis zur Gegenwart, Stuttgart 1936.

27 A. Hettner, Der gegenwärtige Stand der Verkehrsgeographie, in: Geographische Zeitschrift 3, 1897, S. 624-634 und S. 694-704; Vgl. ders., Allgemeine Geographie des Menschen, Bd. 3: Verkehrsgeographie. Hrsg. von Erich Schmitthenner, Stuttgart 1957; ders., Die geographische Verbreitung der Transportmittel dcs Landverkehrs, in: Zeitschrift der Geselschaft tur Voigt, Verkehe (wie Anm.4), Bd. II/2 Die weitere moderne Literatur ubcr Verkehrsgeographie beischungstandes bei E. Otremba/U auf der Heide (Hg.). Handels- und Verkehrsgeographie, in: Wege der Forschung Bd. 363, Darmstadt 1975.
Entwicklung, Methoden und Aufgaben der Verkehrsgeschichte

Bewegung auf, bei der sich dieser seinen Weg auf den Strecken des geringsten Widerstandes sucht, d.h. wie fließendes Wasser Hindernisse prinzipiell zunächst umgeht, statt sie zu durchbrechen. Erst später hat man bei den Geographen erkannt, daß eine rein an der Natur orientierte Betrachtung, welche die politischen, wirtschaftlichen und sozialen Willensakte und damit menschliche Motivationen ausklammert, nicht zur Erklärung ausreicht. Hettner hat, an AJbert Schäffles Überlegungen zur organischen Natur der Gesellschaft sich anlehnend, zu zeigen versucht, daß z.B. ein Wegenetz so gut wie nie einer einzelnen bewußten menschlichen Überlegung entsprang, sondern im Laufe der Generationen historisch gewachsen ist. Als Aufgabe einer Verkehrsgeographie bezeichnete er, die tieferliegenden inneren Gesetzmäßigkeiten solcher Verkehrsausbreitung zu entschleiern, z.B. wanum gewisse Hauptwege des Verkehrs beibehalten und andere wiederum verlegt wurden. Es reiche nicht aus, den Einfluß der natürlichen Umwelt auf die Verkehrsströme oder die wachsende "Verkleinerung der zeit-räumlichen Abstände" zu beschreiben, weil dann die Verkehrsgeographie zu einer reinen "Entfernungswissenschaft" herabsinke. Der eigentliche Gegenstand einer Verkehrsgeographie dürften nicht die sichtbaren Erscheinungen an der Erdoberfläche, d.h. die Verkehrswege sein, sondern die Transporte der Menschen, Güter und Nachrichten. Die Verkehrsgeographie hatte nach Meinung Hettners die Verkehrsströme, die er mit den Blutadern eines Lebewesens vergleicht, aufzuzeichnen und zu erklären. Wenngleich sich Hettner für eine von der Verkehrsgeschichte prinzipiell abgesetzte rein systematisch operierende Verkehrsgeographie einsetzte, konnte er, wie auch schon Karl Ritter in seinem berühmten Aufsatz über das historische Element in der geographischen Wissenschaft 1833 gezeigt hatte, auf die historische Methode nicht verzichten. ${ }^{28}$

Natürlich hat die Entwicklung des modernen Verkehrs, besonders die Anlage von eisernen Brücken, Tunneln, Straßen, Dämmen und Bahnhofshallen, aber auch Schleusen und Hafenbassins, die ebenfalls um diese Zeit emporkommende Technikgeschichte beflügelt. So verfaßte der Österreicher Max Maria Freiherr von Weber eine Reihe höcbst geistvoller Schriften über den Bau von "Sekundär- und Tertiärbahnen" und den Wasserstraßenbau in Nordeuropa, wobei er auf die ältere hydrographische und kulturgeschichtliche Literatur zurückgreifen konnte. ${ }^{29}$ Der Zweig der Verkehrstechnikgeschichte war und blieb ein Faszinosum, das weit über den engeren fachwissenschaftlichen Bereich hinaus Beachtung fand.

\section{Das nachlassende Interesse an verkehrsgeschichtlicher Forschung im $\mathbf{2 0 .}$}

\section{Jahrhundert}

Wenn nicht alles täuscht, dann scheint das lnteresse an der Geschichte des Verkehrswesen seit dem Ersten Weltkrieg spürbar zurückgegangen zu sein. Zwar wurden in der Zei zwischen 1914 und 1945 noch weitere Monographien und Abhandlungen zur Geschichte des Land- und Seetransportes verfaßt, doch blieben die gestellten Ansprüche wie die Ergebnisse

28 K. Ritter, Die Erdkunde im Verhältniß zur Natur und Geschichte des Menschen, 2. erw. Aufh., 10 Bde. Leipzig 1832-1859; ders., Einleitung zu einer vergleichenden Geographie, Leipzig 1852, ders, Aufl., Leipzig 1880.

29 M. M. Frhr. von Weber, Die Wasserstraßen Nord-Europa's Leipzig 1881; ders., Schule des Eisenbahnwesens, 4. Aufl., Leipzig 1885; Vgl. F.M. Feldhaus, Ruhmesblätter der Technik von den Urerfindungen bis zur Gegenwart, Leipzig 1910; ders., Die Technik. Ein Lexikon der Vorzeit, der geschichtlichon Zeit und der Nacurvölker (1914) (unveränderte Neuausgabe München 1970). 
verglichen mit dem 19, Jahhundert relativ bescheiden. Die verstärkte Hinwendung der Wirtschaftswissenschaften $z \mathrm{u}$ rein theoretischen Fragen wirkte sich hier negativ aus. Die wenigen Autoren verfolgten bereits eingeschlagene Bahnen, ohne prinzipiell neue Fragestellungen oder Methoden aufzuwerfen. Walther Vogels "Geschichte der deutschen Seeschiffahrt" (1915), auf frühen Quellenforschungen von Ernst Baasch, Emanuel Fitger und Bernhard Hagedorn basierend, Alfred Birks primár bautechnisch konzipiertes Werk "Die Straße" (1934), ferner Untersuchungen zur Donau- und Rheinschiffahrt, die aber hinter Eberhard Gotheins und Ciristian Eckerts klassischen Studien uber Deutschlands grölten Strom weit zurückblieben, sowie die Festschrift zum einhundertjahrigen Bestehen der deutschen Eisenbahn 1938 verdienen wohl eine Hervorhebung. 33. Nur Anton Felix Napp-Zinn, der später auch einen Handwörterbuchartikel über die Verkehrsgeschichte in Ferdinand Seischabs "Handwörterbuch für Betriebswirtschaftslehre" verfaßte, sowie der Mediavist Walter Stein wagten sich an gröbere Uberblicke heran, ohne aber wie List, Knies, Schnoller, Sax der Sombarl zu wirklich neuen Systematisierungen und generellen Erklärungen vorzustoßen. ${ }^{31}$ Die maßgeblichen Hand und Lehrbücher und die Artikel in den Handwörterbüchern in dieser Periode belegen, wie sehr der ursprünglich so reiche und hoffnungsvolle Ansatz der deutschen Verkehrsgeschichte in der Zwischenkriegszeit verkümmerte. 32

Auch nach dem Zweiten Weltkrieg hat die Verkehrsgeschichte nicht mehr zu den glanzvollen Höhepunkten des späten 19. Jahrhunderts zurückgefunden. So beklagte der eingangs schon erwähnte Nationalokonom Fritz Voigt in seinem 1965 veroffentlichten Werk "Verkehr" die ausgesprochene "Geschichtsfeindlichkeit" seines Faches. In den meisten Lehrbüchern und Monographien würde eine historische Entwicklung der ökonomischen Institutionen völlig vernachlässigt, was er ats Reaktion auf die Theoriefeindlichkeit des Historismus interpretierte. Ohne Zweifel sei das Pendel nun zu weit in die andere Richtung ausgeschlagen, so daß vielfach vergessen werde, die mathematischen Formulierungen und künnen theoretischen Gedankenmodelle empirisch noch zu überprüfen. In seinem großen Werk wollte er zwar keine neuen Beiträge zur Geschichtsforschung liefern, aber doch den Anstob geben, die rein theoretischen Konzeptionen der Verkehrswissenschaft wieder durch

30 W. Vogel, Geschichte der deutschen Seeschiffahra, Bertin 1915; E. Baasch (Ag.), Quelien zur Geschichte won Hamburgs Handel und Schiffahrt im 17, 18. und 19. Jahthundert, Hamburg 1908; E. Figger, Die wirtschaftliche und technische Entwicklung der Seeschiffaht, in: Schriften des Vereins für Socialpolitik 103, Leipzig 1902; B. Hazedom, Die Entwicklung der wichtigsten Schiffstypen bis ins 19. Jahrhundert,

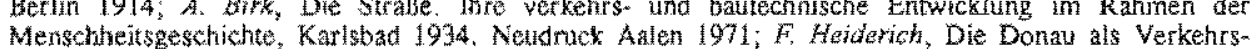

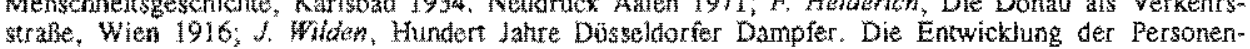
sctiffahrt auf dem Rhein, Koln 1936; $L$. Symphar, Dje Wasserwirtschatt Deutschlands und itre heutigen Aufgaben, 2 Bde, Berlin 1921; Hawpverwaltuing der Deusschen Reichsbahn (HF,), Hundert Jahre devische Eisenbahn, Berlin 1938.

31 AF, Napp-Zinn, Binnenschiffahrt und Eisentahn. Gestalt, Werden und Probleme ihres Verhältnisses im Deurschen Reich, Laiprig 1928; W. Stein, Handels- und Verkentsgeschichte der deutschen Kaiserzeit, Beriin 1922. Bei dem vielzitierten Werk von H. son Sitphan, Geschichte der preuBischen Post, Berlin 1928, handell es sich um die Neuathage seines Werkis Gesstrichte der preufischen Post von ihrem

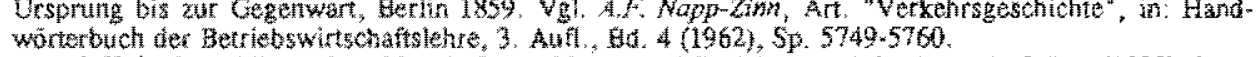
32 Vgl. J.Kulseher, Aligemeine Wirtschaftsgeschichte des Mittelaters und der Neuzeir, 2 Bde.(1928). Neubis zur Gegenwart, 5. verb. Auñ. Leigzig 1928 . eine historische Faktenanalyse zu überprufen. Sein Buch kann daher nicht aur als wichtige Forschungsbilanz, sondern auch als Wendenunkt in der Methodologie angesehen werden

Leider hat Voigts Appell wenig gefruchtet und seine großartige Zusarnmenschau der Verkehrsgeschichte bis heute keine Fortsetzung bei der Histurikerzunft gefunden. Zwar nahm die Wirtschafts- und Sozialgeschichte etwa seit 1960 einen großen institutionellen wie forschungsmäßigen Aufschwung in der Bundesrepublik, doch blieb gerade die Verkehrsgeschichte merkwärdigerweise ein selten beackertes Feld. Die herausragenden Publikationen rnit einem wirklichen Erkenntniszuwachs lassen sich schnell überblicken: Die maritimen Weltgeschichten von Egront Zechlin und Hellnut Diewald, Georg Bessels Geschichte des Norddeutschen Lloyd, Wilhelm Treues reich illustrierter Prachtband uber finflausend Jabre Acbse, Rad und Wagen und Rainer Fremdings höchst innovative Untersuchung uber den Zusammenhang zwischen Eisenbalun und Wirtschaftswachstum, die streng quantifizierend zagieich eine bewuBte Annaherung an die Wirtschaftstheorie sucht, sind als besonders herausragende gelehrte Leistungen zu nennen. ${ }^{3}$

\section{Aufgaben einer "Neuen Verkehrsgeschichte"}

Nach diesem knappen Überblick über Entstehung und Gang der verkehrsgeschichtlichen Untersuchungen ist die Frage zu stellen, welche neuen Forschungsfeldet und Methoden sich für eine sich reaktivierende Verkehrsgeschichte als Subdisziplin der Verketrrswissenschaf ahzeichnen. Solche Überlegungen stellen sich gedanklich verkürzt wie folgt dar:

Zunächst einmal bedürfen die klassischen Felder der Verkehrsgeschichte einer Erweiterung in sachlicher, zeitlicher und xäumlicher Hinsicht. Die bisherigen Monographien und Übersichtsdarstellungen sind hauptsächlich auf bestimmte Themenbereiche wie Eisenbahn, Post und Binnenschiffahrt mit Schwerpunkt in 19. Jahrhundert ausgerichtet. Auch ohne genaue Auszählung dieser weithin verstreuten Titel läßt sich diese, wissenschaftsgeschichtlich allerdings gut erklärbare, einseitige Gewichtung erkennen. Die verstärkte Erforschung des Kraftfahrzeug-, Luftfahrt- und Rohrleitungsverkehrs, aber auch noch des Seeverkehrs, erscheint als ein dringendes Desiderat. Hier gibt es noch überall große Wissenslücken, in die sich allerhand historische Legenden eingenistet haben. So ist es einigermaBen erstaunlich, wie sehr 2.B. die einseitige produktionstechnische Betrachnung des Kraftfahrzeuges seine Fixierung im System der Volkswirtschaft verhindert hat. Die tatsächliche schriftliche Behandlung dieses relativ neuen Verkehrsmittels, za dem bekanntich auch Lastwagen, Omnibusse und Krafträder gehören, durch den Staat im Zusammenhang mit Steuern, Versicherung, Unfälten, Verkehrsregeln, Straßenbau, Spedition und Post, ist erst jetzt durch eine Dissertation von Angela Zatsch an den Tag getreten. ${ }^{34}$

Es gilt überhaupt, nicht nur das einzelne Verkehrsmittel, sondern auch die größere Verkehrsorganisation, die Verkehrsart, die Verkehrsleistungen (Kapazität, Häufigkeit, Geschwindigkeit, Bequemlichkeit, Sicherheit und Kosten) neben den spezifischen

33 E. Zechlin, Maritime Weltgeschichte, Altertum und Minelater, Hambutg 1947; H. Dinwald, Der Kampf un die Weltmeere, Zurich 1980; $G$. Betsat, 1857.1957. Norddeutscher Lloyd Geschichte einer bremischen Reederel, Bremen 1957; W. Tese, Achse, Rad und Wagen. Puntausend Jahre Kulwir- und Technikseschichte, München 1965; R. Framdling, Eisenbahnen und deutsches Wirtschaftswachstum(1840-1879), Dortwund 1975 A. Zautch, Staatsmacht und Motorisienung am Morgen des Industriezeitalters (= Schriften zur Rechts 
Verkehrsbetrieben zu analysieren, um anschließend den ganzen Prozeß einer historischen Umgestaltung ( $z . B$. durch Motorisierung) und dessen Rolle für den Strukhirwandel von Wirtschaft und Gesellschaft deutlich zu machen. So interessant auch die Schilderung technischer Entwicklungen bei einem Verkehrsträger sein mögen, so dürfen diese jedoch nicht im Vordergrund stehen oder gar zum alleinigen Erkenntniszweck werden. Der Blick des Verkehrshistorikers hat stets dem Gesamtzusammenhang zu gelten. Ihn interessieren weniger einzelne Erfindungen, sondern mehr ihre anschließende Verbreitung und vor allem die definitive Durchsetzung in Rahmen einer Volkswirtschaft sowie die sich ergebenden Folgen für die anderen Wirtschaftszweige. Zu einer solchen makrookkonomischen Prozeßanalyse gehört auch immer eine Untersuchung der Widerstände gegen die Einfuhrung von Innovationen und der Handlungsspielräume der jeweiligen Verkehrspolitik.

Un die Bedeutung eines Verkehrsaktes im Sinne einer Raumüberwindung von Personen, Sachgütern und Nachrichten in den geschichtlichen Verlauf richtig einordnen zu können, bedarf es unbedingt einer noch stärkeren Blickerweiterung in frühere Jahrhunderte und einer Lösung vom Zeitalter der Industrialisienung. Die bisherigen Einsichten in das Wesen des Verkehrs sind hăufig allein aus dem Eusenbahnzeitalter abgelesen und so nicht ohne weiteres in andere Perioden und Regionen der Erde ubertragbar. So kannten z.B. Antike, Hoch- und Spätmittelater durchaus schon groBräumige Arbeitsteilungen und einen Fernverkehr, wenngleich der Lokalverkehr natülich bei weitem überwog. Mit Recht ist $z$. B. inzwischen von Wirtschaftshistorikern darauf hingewiesen worden, dals bis zum 19. Jahrhundert eine Kostensenkung durch billigere Frachtkosten keineswegs immer éinen höheren Absatz garantiert hätte. Die Absatzradien wurden häufig durch die hervorragende Qualität einzelner Waren (Snob-Effekt) und nicht durch den Preis bestirnmt.

In den älteren Verkehrsgeschichten, deren Verfasser oft durch die liberale Fortschrittsbrille des 19. Jahrhunderts sahen, wurden die vorindustriellen Verkehrsmittel oftmals als primitiv herabgewüdigt. Dabei wurde übersehen, daß viele altere Verkehrsmittel und Verkehrsorganisationen fur die Ansprüche ihrer Zeit durchaus optimal waren. Reitende Boten, Marktschiffe, Flobe, Sänften und Kutschen waren aus trüherer Sicht vielfach durchaus okonomisch effizient. Es ist bezeichnend fü dis kurzichtige moderne Techniksicht und den urangebrachten Fortschrittsoptimisnus, daß es bis beute keine wissenschaftlich brauchbare Monographie uber die Geschichte des Pferdes und des Fahrrades als Verkehrsmittel, geschweige denn des Fufgängers gibt, obwohl alle diese Formen der Raumberwindung lange eine mehr oder weniger grofie Rolle gespielt haben und in manchen

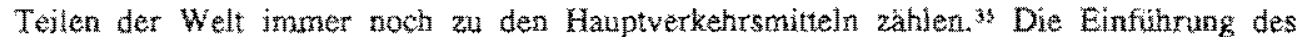

35 Die Literatur wher Pferde und Reiten beschäftigt sich fast ausschlieblich mit Pferdezuch, Pferderassen, Pferdiesport und dort besonders mit Turnieren, Dressur und Rejtschulen, dann aber auch mit der

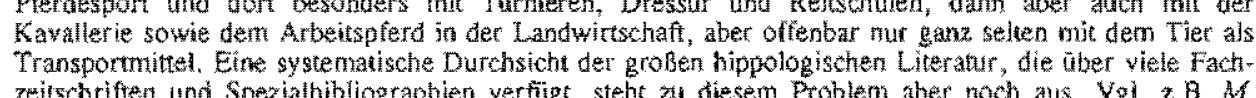
zeitschriften und Spezialbibliographien verfigt, steht zu diesem Problem aber noch aus. Vgi. Z. B. M. Jahth, RoB und Reiter in Leben und Sprache, Glauben und Geschichte der Deurschen, 2 Bde, Leipzig
1872 ; Graf $K G$. Wrangel, Das Buch der Pferde. Ein Handbuch fuir jeden Besitzer und Liebhaber von Pferden, 2 Bde, 6. Aufl, Stuttgart 19271928, L, von Heydebrand und der Lasa, Xllustrirte Geschichte der Reiterei. Die Entwicklung der Reitkunse seit Jahrtausenden, Wien 1892; H. Mollew L Bom, Handbuch der Pferdekunde. Für Ofiziere und Landwirte, 6.Aufl., Berlin 1910. Auch die Geschichte des Fahtrades und Radtahrens ist erst enseitig erforscht und fast ausnahmslos auf dite Entwicklung des Radsports und
Entwicklung, Wethoden und Aufgaben der Verkehrsgeschichte

Falrrades am Ende des 19. Jahrhunderts, das nach erstaunlich kurzer Zeit von Millionen Menschen benutzt wurde, war zumindest eine kleine Verkehrsrevolution, da hier erstmals dem Menschen die Möglichkeit geboten wurde, sich auf vergleichsweise billige Weise unabhängig vom starren Linienverkehr eine private räumliche Mobilităt zu schaffen. Auch heute ist das Fahrrad neben den eigenen Füßen das wohl am meisten benutzte Fortbewegungsmittel auf der ganzen Welt. Vergleiche mit anderen Epochen und Ländern vernögen hier den Blick des Verkehrshistorikers ungemein zu schärfen.

Natürlich sollte eine künftige Verkehrsgeschichte im Zeichen der zunehmenden europä:schen Integration auch versuchen, über die rein nationale Blickweise hinaus zu gelangen: Die bisherigen Wirtschaftsgeschichten mit einem europäischen Anspruch bieiben freilich noch frustrierend, handelt es sich doch noch um reine Additionen nationaler Verkehrsgeschichten, die micht unter einheitlichen Fragestellungen zu einem größeren Gemälde zusammengesehen wurden. Ohne die Vereinheitlichung der Statistiken lassen sich $z$.B. nur wenige internationale Generalisierungen machen. ${ }^{36}$ Mit Ausnahme der Schiffahrt sind die grenzuberschreitenden Verkehrstrome erst bruchstückhaft erfaft. Fritz Voigt hat in seinem groben Werk gezeigt, welche Statistiken für den Binnen- und Außenverkehr zur Messung des Transports von Gütern, Personen und Nachrichten zur Verfügung stehen. ${ }^{37}$ Aber die Füle der Territorialstatistiken vor der Reichsgrundung ist von ihm noch micht berücksichtigt worden. Auf jeden Fall Jassen sich Eisenbahn- und Schiffsverkehr, bei dern der Staat aus hoheittichen und steuerlichen Günden frühzeitig Daten sammelte, sehr viel besser statistisch erfassen als der Landtransport, der sich insgesamt mehr im privaten Sektor abspieite. Im Rahmen eines Schwerpunktprogramms der Deutschen Forschungsgemeinschaft haben die beiden Wirtschaftshistorker Rainer Fremdling und Andreas Kunz, alle verfügbaren gedruckten Quellen aufarbeitend, damit begonnen, erstmals verläßliche Langzeitreihen für den Nak-, Eisenbahn-, See und Binnenschiffsverkehr in 19. und 20. Jahrhundert zu rekonstruieren. Alle älteren, viel zu groben, methodisch noch unzuverlässigen Verkehrs. statistiken dürten dann hinfallig werden, auch die Verkebrszahen in Walter $G$. Hoffmanns bekanntem Kompendium "Das Wachstum der deutschen Wirtschaft seit der Mitte des 19.

L. Croon, Das Fatrrad und seine Entwikklung, Berlin 1939; M.J.B. Rauth ua, Mit dem Rad dureh zwei Jahrhunderte. Das Fahtrad und seine Geschichte, Aaral 1979, F, A. Pawri, Die Geschichte des Fahrrades,

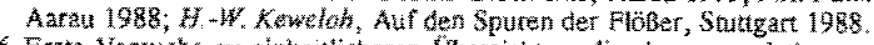

36 Erste Versuche zu eimhenticheren thersichten, die aber nur relativ wenige Länder berücksichtigen, sind z.B. CM. Cipollat $u, a$, Europaische Wirtschaftsgeschichte. The Fontana Economic history of Europe, 5 Bde, Stuttgart 1953, A.S. AthwardS.B. Pöst, The Economic Development of Continental Europe, 1780Europe, $1830-1914$, Landon 1982; SP. Vilie. Transport and the Development of Europan Fconomy $1750-1918$, London 1990 . Vgl. als einschlsgige wichtige Statistik, die auch den Verketh benomy, berucksichrigt, $R, B$. Witchell, European Historical Statistics, 1750-1975, 2. Aufl, London 1981.

37 Ygl. Voigt, Verkehr (wie Anm,4), Bd. M/2(1965), S.1280-1284.

38 W. Fischer/A. Kumz, Grundlagen der historischen Statistik in Deutschland. Quellen, Methoden, Forschungsziele, Opladen $1991 ;$ R. Fremdling/A. Kunz, Historische Verkehrsstatiscik von Deutschland, in: N. Diederich Ha. $\left(\mathrm{Hg}_{\mathrm{g}}\right)$, Historische Statistik in der Bundesrepublik Deutschland, in: Fonsm der Bundesstatistix Bd. 15, Stuttgarn 1991, \$ 90106; A. Kunz, Database Applications in Economic History, in: H. Best exal. (eds.). Computers in the Humanities and Social Sciences, Achievements of the 1980 : Prospects for the 1990s. Proceedings of the Cologne Computer Conference 1988: Use of the Computers in the Humanities and Social Sciences theid at the University of Cologne, September 1988, Munchen 1991. Ein Datenhandbuch, wird spater die Statistik der Essenbalinen, der See und Binnensehiffahrt sowie in Deutschland 1835-1989 (= Historische Verketrsstatistik von Deutschland, Bd. 1), St Katharinen 199 \% 
Jahrhunderts". ${ }^{39}$ Der Bonner Historiker Hans Pohl hat außerdem erst kürzlich die Zahlen für den Weltverkehr und die wichtigsten Innovationen zwischen der Mitte des 19. Jahrhunderts und 1914 zusammengefaßt, eine Studie, die die höchst ungleichmäßige Aufhellung der Transportgeschichte in den einzelnen Ländern noch einmal deutlich macht. ${ }^{40}$

Es ist an dieser Stelle unmöglich, auf alle Problembereiche einer Verkehrsgeschichte einzugehen. Fritz Voigt hat diese bereits in gedankenreicher Weise charakterisiert: Es ist generell nach den Antrieben für eine eigenständige Entwicklungsdynamik, d.h. den Impulsen für den Ausbau der verschiedenen Techniken, Organisationen und Unternehmen des Verkehrs sowie nach der Dichte von Verkehrsnetzen und ihren internen wie externen Determinanten und den Verschiebungen der Schwerpunkte zu fragen. Im Mittelpunkt der Betrachtungen hat dann der Beitrag des Verkehrs zum Wirtschaftswachstum zu stehen. Dabei ist aber nicht nur an den vielzitierten Prozeß der Industrialisierung, sondern auch an die Fragen der Verstädterung sowie der gesamten soziokulturellen Modernisierung des Lebens zu denken. Die Entleerung gewisser Regionen durch den Verkehr ist historisch ebenso folgenreich gewesen wie die Zusammenballung der Menschen an anderen Orten. Der Einfluß von Eisenbahn und Kanalbau auf das städtische Bevölkerungswachstum und den gewerblichen Ausbau ist erst wenig systematisch durchleuchtet worden. Hier werden alte Probleme der Standorttheorie berührt, die seit Johann Heinrich von Thünen die Volkswirte immer wieder beschäftigt haben. Die Differenzierung der Produkte und ihr Absatz als Folge neuer Verkehrswege und gewachsener Verkehrsschnelligkeit sind neuerdings wieder Gegenstand der Konsumtionsgeschichte geworden. ${ }^{41}$

Besondere Beachtung verdient auch die bisher hier nur kurz erwähnte Geschichte des Nachrichtenverkehrs. Da wir seit der massenhaften Verbreitung von Telefon, Rundfunk, Fernsehen und elektronischer Datenfernübertragung (Telex, Telefax, Digitaltelefon, Zusammenfassung digitaler Datenbanken zu einem integrierten Fernmeldenetz) erkennbar in ein ganz neues Zeitalter der Massenkommunikation eingetreten sind, fühlten sich die Wirtschafts- und Sozialhistoriker vor einiger Zeit veranlaßt, ihren Wissensstand auf diesem Gebiet einmal zusammenzufassen. ${ }^{42}$ Hier hat sich zunächst gezeigt, daß der heute so inflationär benutzte Begriff "Kommunikation" einen entscheidenden Bedeutungswandel durchgemacht hat. Wurde der Ausdruck im 19. Jahrhundert unter dem Eindruck des neuen Verkehrsmittels noch als Synonym für Verkehr bzw. Mittel zur Beförderung von Sachen, Personen und Informationen gebraucht, so verstand man später eingeschränkt im englischen Sprachbereich darunter nur das Fernmeldewesen im engeren Sinne, was sich dann international durchsetzte. Im 20. Jahrhundert wurde Kommunikation in den Sozialwissenschaften

39 W.G. Hoffmann, Das Wachstum der deutschen Wirtschaft seit der Mitte des 19. Jahrhunderts, Berlin 1965. Vgl. H. Kelterer, Verkehrsstatistik, Berlin 1936; O. Schlier, Verkehrsstatistik, in: Statistik in Deutschland, Berlin 1940; H. Rogmann, Deutscher Verkehr in graphischer Darstellung, Düsseldorf 1952;
I. Esemwein-Rothe, Art. "Verkehrsstatistik", in: Handwörterbuch der Sozialwissenschaften Bd. 11, Stuttgart 1960, S. $137-141$.

$40 \mathrm{H}$. Pohl, Auforuch zur Weltwirtschaft. Geschichte der Weltwirtschaft von der Mitte des 19. Jahrhunderts bis zum Ersten Weltkrieg, Stuttgart 1989, S. 213-245

41 H.J. Teuteberg $(H g$.$) , Durchbruch zum Massenkonsum. Lebensmittelmärkte und Lebensmittelqualität im$ Städtewachstum des Industriezeitalters, Münster 1987; A. Kunz, Die Verknüpfung von Märkten und Transport. Verkehrsstatistik und Marktintegration in Agrarregionen, in. Ti Piorer

$42 \mathrm{H}$. Pohl (Hg.), Die Bedeutung der Kommunikation für Wirtschaft und Gesellschaft, Stuttgart 1989.
Entwicklung, Methoden und Aufgaben der Verkehrsgeschichte

dann als Erfahrungsweitergabe und schließlich sogar als Medium zur Ausübung von Herrschaft interpretiert. ${ }^{43}$

Wenngleich Wirtschaftswissenschaftler, Geographen und Historiker den Begriff weiterhin unter dem Oberbegriff Verkehr subsumierten und entsprechend eng gefaßte Untersuchungen betrieben, so fächerte sich der Terminus im allgemeinen Sprachgebrauch doch immer weiter auf, so daß er heute zahlreiche Komplexe im wirtschaftlichen und sozialen Bereich erfaßt und relativ diffus geworden ist. Mit dem Kommunikationswesen beschäftigen sich inzwischen nicht nur Ökonomen, sondern auch Psychologen, Soziologen, Publizisten, Pädagogen, Anthropologen usw. Sie haben zum Teil ihre eigenen Definitionen und theoretischen Rahmenkonzepte entwickelt, so daß eine Verständigung schwierig geworden ist. Die Verkehrshistoriker müssen daher klären, welche Formen der Raumüberwindung, soziokulturellen Vermittlung und menschlichen Verständigung sie untersuchen wollen. Es scheint ratsam, auch hier zwischen einer engeren wirtschaftlich orientierten und einer weiteren kulturell ausgerichteten Kommunikation zu differenzieren. Die inzwischen weit entwickelten Kommunikations- und Informationstheorien, die vielfach auf mathematischkybernetischen Modellen beruhen, müssen auf ihre historische Anwendbarkeit überprüft werden. ${ }^{44}$ Es ist dabei die Frage aufzuwerfen, ob der sich immer schneller ausdifferenzierende Wissenszweig der Kommunikationsforschung künftig überhaupt noch zur Verkehrswissenschaft und zur Verkehrsgeschichte gerechnet werden kann, da diese inzwischen so intensiv von Nachbardisziplinen betrieben wird

Die Bilanz der Historiker zum Thema "Die Bedeutung der Kommunikation für Wirtschaft und Gesellschaft" hat ferner darauf aufmerksam gemacht, daß die Geschichte des Reisens zu den zentralen Sektoren einer historisch orientierten Verkehrswissenschaft gerechnet werden muß. Nicht nur das Aufkommen des Massentourismus etwa seit 1960, der heute ein bedeutender Wirtschaftszweig geworden ist, sondern auch die älteren Formen dieser personalen Raumüberwindung bedürfen einer intensiven Erforschung. Die inzwischen auch international vergleichend betriebene historische Reiseforschung, deren Ergiebigkeit und Methoden früher ganz falsch eingeschätzt wurden, hat inzwischen erstaunliche Fortschritte gemacht und wird sogar. von verschiedenen Diszipiinen gleichzeitig betrieben. ${ }^{45} \mathrm{lhr}$ Problemfeld ist freilich noch schwer abzugrenzen, da seit antiker Zeit Schriftsteller über das Reisen zu Lande und zu Wasser regelmäßig berichtet haben. Sie produzierten eine vorläufig nicht $z u$ übersehende Zahl von Reiseführern und Reiseberichten, die erst zu einem geringen Teil ausgewertet wurden. In dem vielbändigen Quellenmaterial, das in jeder größeren

43 H. Beth/H. Pross, Einführung in die Kommunikationswissenschaft, Stuttgart 1976; F. Naschold, Kommunikationstheorien, in: J. Aufermann/H. Borgmann/R. Sülz (Hg.), Gesellschaftliche Kommunikation und Neuwied 1962 .

44 N. Wiener, Kybernetik, Düsseldorf 1963; C.E. Shannon/W. Weaver, The Mathematical Theory of Conımunication, Urbana, Illinois 1949; K. Steinbach, Techniken der Kommunikation, in: O. Haseloff (Hg.), Forschung und Information, 2.Aufl., Bd. 3, Berlin 1971, S. 32ff.; O.H. Poensgen, Art. "Kommunikation", in: Handwörterbuch der Wirtschaftswissenschaft Bd. 4, Stuttgart 1978, Sp.466-477. 45 A. Maçzak/H.J. Teuteberg (Hg.), Reiseberichte als Quellen europäischer Kulturgeschichte. Aufgaben und Möglichkeiten der historischen Reiseforschung, Wolfenbüttel 1982; B.I. Krasnobaev u.a., Reisen und Reisebeschreibungen im 18. und 19. Jahrhundert als Quellen der Kulturbeziehungsforschung, Essen 1987; B.W. Bathe, Von den Kreuzzügen zu den Kreuzfahrten, Bielefeld 1972; N. Ohler, Reisen im Kommunikation (wie Anm.39), S. 216.255. 
Bibliothek $z u$ finden ist, werden nicht nur genaue Angaben uber Reiserouten und Enffernungen, den Zustand der Wege und de Verkelrserleichterungen (Bricken, Pässe, Hospize ete.), sondern auch uber das jeweillge Verkehrsrecht genacht. Die historische Reiseforschung berührt so gut wie alle Zweige der übrigen Geschichtswissenschaft.

Die überlieferten Reiseberichte schildern wie keine andere historische Quelle überdies die subjektiven Eindrücke desjenigen, dex eine Ortsverändarung vornahm. Dieser Aspekt ist; soweit man erkennen kann, von der bisherigen Verkehrsgeschichte nut partiell erfaßt worden. Bis heute wissen wir nur wenig Zusammenhängendes, wie z.B. ein reisender Kaiser in Mittelalter, ein wandernder Handwerksgeselte in 16. Jahıhundert, der Insasse einer Postkutsche um 1800 oder die Auswandereffamilie auf einem Schiff zwischen Bremen und New York um 1850 die Raumüberwindung erlebt haben. Der persönliche Erfahrungshorizont ist aber ein wichtiger historischer Gegenstand, da er ganz dicht an das zu beobachtende historische Ereignis Verkehrr heranführt.

Wolfgang Schivelbusch hat in seiner vielbeachteten "Geschichte der Eisenbahnreise" (1977) gezeigt, welchen Stellenwert dies neue Verkehrsmittel im Bewuitsein der Menschen einzunehmen begann und tausendjährige Vorstellungen vom Raum-Zeit-Verhältnis damals grundsätalich neu formiert wurden. ${ }^{46}$ Seit dem Beginn der großen Verkehrsrevolution im 19. Jahrhundert wurde nach hhm immer mehr die Vorstellung populär, daß "Circulation", d.h. Kommunikation, Austausch und Bewegung, den Fortschritt und die Aufklärung der Menschheit bedeuten, wogegen Sebhaftigkeit und Abgeschlossenheit als rückstãndig zu überwinden seien. Bürgerliche Kulturanschauung und Verkehrsaufschwung sind damals Hand in Hand gegangen und haben so zum gesellschaftichen Wandel beigetragen. Unter anderem hat die Eisenbahn den Sinn der alten Bildungsreise, die jahrhundertelang einen festen Platz im Kulturleben hatte, weitgehend aufgehoben und dem wachsenden Tourismusangebot einen Warencharakter verliehen. Die darauf aufbauende Kulturkritik hat freilich dabei übersehen, daß die Massernhaftigkeit des Reisens auch eine nie dagewesene neue soziale Emanzipation bractite. Peter Borscheid, früher in Münster und nun in Marburg Wirtschafts- und Sozialgeschichte lehrend, hat am Beispiel des Automobils gezeigt, wie sich durch seine masserhafte Verbreitung das ursprünglich kleinräumliche Muster der Mobilität auflöste und die Motorisierungswelle tiefeingeschliffene Alltagsgewohnheiten der Menschen von Grund auf veränderte und z,B. zu einer "tertiären Verstädterung" geführt hat ${ }^{47}$ Der massenhafte Autoverkehr brachte auf der einen Seite neue soziale Isolation im Verkehr, aber auch andererseits eine neue Integrierung; so sind die Jahthundertealten Stadt-Land-Unterschiede erst durch ihn völlig eingeebnet worden. Die gesellschaftichen und wirtschaftichen Kosten der explodierenden Mobilität bedeuten einen Themenkomplex, der uns erst in den letzten Jahrzehnten voll bewuBt geworden ist. Bei der Lösung der brennenden Probleme des Nahverkebrs aber auch des Wettbewerbs der Verkehrsträger kann die Verkehrsgeschichte wertvolle Hilfe leisten, da diese Fragen zum Tell schon seit einem Jahrhundert diskutiert werden.

46 W. Schivelbusch, Geschichte der Eisenbahnreise. Zur Industrialisierung von Raum und Zeiz im 19. Jahrlsundert, Münctien 1977 .

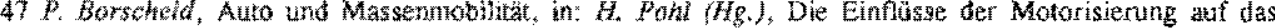
Verkestrswesen von 1886 bis 1986 , Suttigat 1988 , S. 117-141. 